\title{
On the set of numbers $\{14,22,30,42,90\}$
}

\author{
by
}

VAmsi K. Моотна (Boston, Mass.)

For a fixed integer $t$, a size $n P_{t^{-}}$set is a set $\left\{q_{1}, \ldots, q_{n}\right\}$ of distinct positive integers such that $q_{i} q_{j}+t$ is the square of an integer whenever $i \neq j$. For example, $\{1,2,5\}$ is a $P_{-1}$-set, while $\{1,3,8,120\}$ is a size 4 $P_{1}$-set. A $P_{t}$-set $S$ is extendible if there exists a positive integer $d \notin S$ such that $S \cup\{d\}$ is still a $P_{t}$-set.

Problems related to $P_{t}$-sets date back to the time of Diophantus (see Dickson [4, Vol. II, p. 513]). The most famous recent result is in the area of extending $P_{t}$-sets and is due to Baker and Davenport [1], who used Diophantine approximation to show that the $P_{1}$-set $\{1,3,8,120\}$ is nonextendible. Other methods for arriving at the same result were subsequently described (Kanagasabapathy and Ponnudurai [6], Sansone [9], and Grinstead [5]). Several more recent papers have made efforts to characterize the extendibility of classes of $P_{t}$-sets (Brown [3], Mootha and Berzsenyi [7]).

In this paper we introduce a very simple method for assessing the extendibility of $P_{t}$-sets of the form $\{a, b, a k, b k\}$, where $a, b$, and $k$ are integers. The technique is illustrated by demonstrating the nonextendibility of the first identified size $5 P_{t}$-set (see Berzsenyi [2]):

Theorem. The $P_{-299}$-set $\{14,22,30,42,90\}$ is nonextendible.

Proof. First, note that if we set $a=14, b=30$, and $k=3$, then this set is of the form $\{a, b, a k, b k, 22\}$. Showing that this $P_{t}$-set is nonextendible is equivalent to showing that the system of equations

$$
\left\{\begin{array}{l}
14 d-299=w^{2} \\
30 d-299=x^{2} \\
42 d-299=y^{2} \\
90 d-299=z^{2}
\end{array}\right.
$$

has exactly one integer solution, $d=22$, which corresponds to the fifth member of the $P_{-299}$-set. Eliminating $d$ between $(*)$, we obtain the following

1991 Mathematics Subject Classification: Primary 11D09; Secondary 11B37. 
Pellian equations:

$$
\left\{\begin{array}{c}
y^{2}-3 w^{2}=598 \\
z^{2}-3 x^{2}=598
\end{array}\right.
$$

This is a system of two Pellian equations, each having exactly four classes of solutions (see Nagell [8, p. 205]) given by

$$
\begin{aligned}
& \mathbf{K}_{1}: y_{n}+\sqrt{3} w_{n}=z_{n}+\sqrt{3} x_{n}=(25+3 \sqrt{3})(2+\sqrt{3})^{n}, \\
& \overline{\mathbf{K}}_{1}: y_{n}+\sqrt{3} w_{n}=z_{n}+\sqrt{3} x_{n}=(25-3 \sqrt{3})(2+\sqrt{3})^{n}, \\
& \mathbf{K}_{2}: y_{n}+\sqrt{3} w_{n}=z_{n}+\sqrt{3} x_{n}=(29+9 \sqrt{3})(2+\sqrt{3})^{n}, \\
& \overline{\mathbf{K}}_{2}: y_{n}+\sqrt{3} w_{n}=z_{n}+\sqrt{3} x_{n}=(29-9 \sqrt{3})(2+\sqrt{3})^{n},
\end{aligned}
$$

where $n$ is a whole number. These solutions correspond to the linear recurrent sequence $w_{n}=4 w_{n-1}-w_{n-2}, n \geq 2$, where $w_{0}$ and $w_{1}$ depend on the solution class (and similarly for $x_{n}$ ). Using recurrence relations, we produce explicit expressions for each of the four solution classes:

(2)

$$
\left\{\begin{array}{l}
\mathbf{K}_{1}: w_{n}=x_{n}=\left(\frac{9+25 \sqrt{3}}{6}\right)(2+\sqrt{3})^{n}+\left(\frac{9-25 \sqrt{3}}{6}\right)(2-\sqrt{3})^{n}, \\
\overline{\mathbf{K}}_{1}: w_{n}=x_{n}=\left(\frac{9-25 \sqrt{3}}{-6}\right)(2+\sqrt{3})^{n}+\left(\frac{9+25 \sqrt{3}}{-6}\right)(2-\sqrt{3})^{n}, \\
\mathbf{K}_{2}: w_{n}=x_{n}=\left(\frac{27+29 \sqrt{3}}{6}\right)(2+\sqrt{3})^{n}+\left(\frac{27-29 \sqrt{3}}{6}\right)(2-\sqrt{3})^{n}, \\
\overline{\mathbf{K}}_{2}: w_{n}=x_{n}=\left(\frac{27-29 \sqrt{3}}{-6}\right)(2+\sqrt{3})^{n}+\left(\frac{27+29 \sqrt{3}}{-6}\right)(2-\sqrt{3})^{n} .
\end{array}\right.
$$

Table 1 is a list of the first 9 solutions $w_{n}=x_{n}$ in each of the four classes.

Table 1. Some solutions $w_{n}$ and $x_{n}$

\begin{tabular}{|lllll|}
\hline$n$ & $w_{n}=x_{n} \in \mathbf{K}_{1}$ & $w_{n}=x_{n} \in \overline{\mathbf{K}}_{1}$ & $w_{n}=x_{n} \in \mathbf{K}_{2}$ & $w_{n}=x_{n} \in \overline{\mathbf{K}}_{2}$ \\
\hline 0 & 3 & -3 & 9 & -9 \\
1 & 31 & 19 & 47 & 11 \\
2 & 121 & 79 & 179 & 53 \\
3 & 453 & 297 & 669 & 201 \\
4 & 1691 & 1109 & 2499 & 751 \\
5 & 6311 & 4139 & 9319 & 2803 \\
6 & 23553 & 15447 & 34779 & 10461 \\
7 & 87901 & 57649 & 129898 & 39041 \\
8 & 328051 & 215149 & 484409 & 145703 \\
\hline
\end{tabular}


Because we have derived closed expressions for $w_{n}$ and $x_{n}$, we can set $w=w_{j}$ and $x=x_{i}$, for some whole numbers $i$ and $j$. From $(*)$, it becomes clear that since $x^{2} / w^{2}=x_{i}^{2} / w_{j}^{2}=(30 d-299) /(14 d-299)$,

$$
\frac{x_{i}}{w_{j}} \approx \sqrt{\frac{15}{7}}=1.4638501 \ldots \text { for large } d .
$$

This provides us with an additional constraint which must be satisfied simultaneously with (1) for sufficiently large values of $d$. Hence, if there is an integer $d \neq 22$ that solves $(*)$, and $d$ is large, then we expect $x_{i} / w_{j}$ to be asymptotically equal to $1.4638501 \ldots$ For computational purposes, it is necessary to formalize what we mean by "sufficiently large" values of $d$. We define

$$
\varepsilon(d) \equiv\left|\sqrt{\frac{30 d-299}{14 d-299}}-\sqrt{\frac{15}{7}}\right|=\left|\frac{x_{i}}{w_{j}}-\sqrt{\frac{15}{7}}\right|
$$

and note that $\varepsilon(d) \rightarrow 0$ as $d \rightarrow \infty$. In particular, observe that for $d \geq$ $8.34 \times 10^{8}$ (i.e., $w_{j} \geq 1.08 \times 10^{5}$ and $x_{i} \geq 1.58 \times 10^{5}$ ) we must have $\varepsilon(d) \leq 10^{-8}$. Table 1 lists all values of $x_{i} \leq 1.58 \times 10^{5}$, and simple trial and error of these values indicates that the only solution in this range corresponds to $d=22$. Hence, $x_{i}$ and $w_{j}$ must be so large that $d \geq 8.34 \times 10^{8}$ and $\varepsilon(d) \leq 10^{-8}$.

We now demonstrate that no selection of large $x_{i}$ and $w_{j}$ (i.e., $x_{i} \geq$ $1.58 \times 10^{5}$ and $\left.w_{j} \geq 1.08 \times 10^{5}\right)$ meets this requirement. By selection, we mean a choice of two classes from which to assign values to $x$ and $w$, e.g., $x=x_{i} \in \mathbf{K}_{1}$ and $w=w_{j} \in \overline{\mathbf{K}}_{2}$, or $x=x_{i} \in \mathbf{K}_{2}$ and $w=w_{j} \in \overline{\mathbf{K}}_{2}$, etc. Clearly, there are a total of 16 possible selections that we must consider, and we treat each case separately:

Case 1: $x=x_{i} \in \mathbf{K}_{1}$ and $w=w_{j} \in \mathbf{K}_{1}$. From $(*)$, we see that $x>w$, which implies that $i>j$. We must attempt to minimize $\varepsilon(d)$, and the best we can do is to choose $i=j+1$, implying that $x / w=w_{j+1} / w_{j}$. From (2), we find that $\varepsilon(d)$ decreases monotonically for increasing $d$. But

$$
\lim _{d \rightarrow \infty} \varepsilon(d)=\lim _{j \rightarrow \infty}\left|\frac{w_{j+1}}{w_{j}}-\sqrt{\frac{15}{7}}\right|=2.2682006 \ldots \gg 10^{-8} .
$$

Hence, selecting both $x$ and $w$ from $\mathbf{K}_{1}$ cannot satisfy $(*)$ for large values of $d$.

Case 2: $x=x_{i} \in \overline{\mathbf{K}}_{1}$ and $w=w_{j} \in \mathbf{K}_{1}$. Again, because $x>w$, we are forced to choose $i=j+1$ to minimize $\varepsilon(d)$. $\varepsilon(d)$ decreases monotonically with increasing $d$, and we find from (2) that 


$$
\lim _{d \rightarrow \infty} \varepsilon(d)=\lim _{j \rightarrow \infty}\left|\frac{x_{j+1}}{w_{j}}-\sqrt{\frac{15}{7}}\right|=0.9837784 \ldots \gg 10^{-8} .
$$

Conclude that this particular selection of $x$ and $w$ does not yield a large solution to $(*)$.

The remaining fourteen cases are treated similarly, and the results are summarized in Table 2. For each selection, the "best" index choice (which minimizes $\varepsilon(d))$ and $M=\lim _{d \rightarrow \infty} \varepsilon(d)$ are shown.

Table 2. Summary of 16 cases

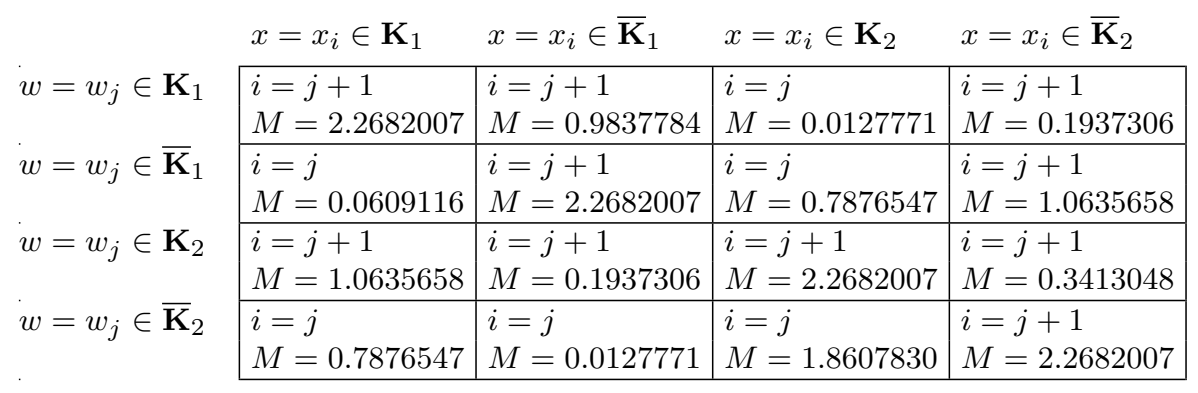

Note that in all cases, $\lim _{d \rightarrow \infty} \varepsilon(d)$ is much greater than $10^{-8}$, which means that we have safely precluded the possibility of a "large" solution to (*).

As we have already exhausted all possibilities in Table 1, we conclude that the $P_{-299}$-set $\{14,22,30,42,90\}$ is nonextendible.

This same approach can be taken in quickly assessing the extendibility of any $P_{t}$-set of the form $\{a, b, a k, b k\}$.

Acknowledgement. I am most grateful to George Berzsenyi, who introduced me to the problem of $P_{t}$-sets and motivated this research.

\section{References}

[1] A. Baker and H. Davenport, The equations $3 x^{2}-2=y^{2}$ and $8 x^{2}-7=z^{2}$, Quart. J. Math. 20 (1969), 129-137.

[2] G. Berzsenyi, Adventures among $P_{t}$-sets, Quantum 1 (1991), 57.

[3] E. Brown, Sets in which $x y+k$ is always a square, Math. Comp. 45 (1985), 613-620.

[4] L. E. Dickson, History of the Theory of Numbers, Vol. II, Carnegie Institution, Washington, 1920; reprinted, Chelsea, New York, 1966.

[5] C. M. Grinstead, On a method of solving a class of diophantine equations, Math. Comp. 32 (1978), 936-940.

[6] P. Kanagasabapathy and T. Ponnudurai, The simultaneous diophantine equations $y^{2}-3 x^{2}=-2$ and $z^{2}-8 x^{2}=-7$, Quart. J. Math. 26 (1975), 275-278.

[7] V. Mootha and G. Berzsenyi, Characterizations and extendibility of $P_{t}$-sets, Fibonacci Quart. 27 (1989), 287-288. 
[8] T. Nagell, Introduction to Number Theory, Wiley, New York, 1951.

[9] G. Sansone, Il sistema diofanteo $N+1=x^{2}, 3 N+1=y^{2}, 8 N+1=z^{2}$, Ann. Mat. Pura Appl. 111 (1976), 125-151.

DIVISION OF HEALTH SCIENCES AND TECHNOLOGY

HARVARD MEDICAL SCHOOL

BOSTON, MASSACHUSETTS 02115

U.S.A. 